\title{
Selecting Adjuvant Treatment for Endometrial Carcinoma Using Molecular Risk Factors
}

\author{
Bastiaan G. Wortman ${ }^{1} \cdot$ Remi A. Nout ${ }^{1} \cdot$ Tjalling Bosse $^{2} \cdot$ Carien L. Creutzberg $^{1}$
}

Published online: 31 July 2019

(C) The Author(s) 2019

\begin{abstract}
Purpose of Review To provide an overview of common molecular risk factors in endometrial cancer (EC) with the possibility to improve adjuvant treatment selection.

Recent Findings Recent studies have discovered and confirmed four different molecular subclasses in EC, with each having a distinct prognosis; POLE-ultramutated, microsatellite unstable, copy-number low, and copy-number high. Subsequent studies have shown that combining both molecular with clinicopathological risk factors can potentially improve adjuvant treatment selection for women with high-intermediate risk EC. For high risk and advanced stage EC, several molecular alterations are being explored for targeted therapy.

Summary Molecular alterations are frequently found in endometrial cancer and have currently not been implemented in the treatment guidelines for EC. Assessment of molecular alterations can distinguish patients that require less or more intensified adjuvant treatment. Trials investigating targeted therapies in EC are ongoing and have shown some promising results, however, more evidence is needed and results of randomized trials have to be awaited.
\end{abstract}

Keywords Endometrial cancer $\cdot$ Adjuvant treatment $\cdot$ Radiotherapy $\cdot$ Chemotherapy $\cdot$ Molecular risk factors $\cdot$ Molecular alterations $\cdot$ Targeted therapies $\cdot$ Checkpoint inhibitors $\cdot$ PARP inhibitors

\section{Introduction}

\section{Epidemiology}

Endometrial cancer (EC) is the most common gynecological cancer in postmenopausal women in developed countries and the incidence is rising due to increased obesity and aging of the population. In the US, endometrial cancer is the 4th most common cancer in women with an estimated incidence of 61,880 new cases in 2019. Most women with EC are diagnosed between the ages of 60 and 80, and most are diagnosed at early stage of disease due to early symptoms such as vaginal

This article is part of the Topical Collection on Gynecologic Cancers

Bastiaan G. Wortman

b.g.wortman@lumc.nl

1 Department of Radiation Oncology, Leiden University Medical Center, K1-P, Albinusdreef 2, P.O. Box 9600, 2300 RC Leiden, The Netherlands

2 Department of Pathology, Leiden University Medical Center, Leiden, The Netherlands bleeding. This generally results in a favorable prognosis and a relatively low number of cancer-related deaths (estimated 12,160 in 2019 in the US) $[1,2]$.

\section{Histology and Risk Factors}

The most common histological type of EC is endometrioid adenocarcinoma (EEC), accounting for approximately $70-80 \%$ of EC. Non-endometrioid cancers (NEEC) mainly comprise serous and clear cell cancers, accounting for approximately $5-10 \%$ and $1-5 \%$ of EC, respectively, and the other aggressive subtypes are undifferentiated and dedifferentiated EC and uterine carcinosarcomas. Well-established clinicopathological risk factors, used in the current treatment guidelines, are age, histological type, tumor grade, International Federation of Gynecology and Obstetrics (FIGO)-stage, depth of myometrial invasion, and presence and extent of lymph-vascular space invasion (LVSI). EEC is graded using the FIGO grading system as low grade (grade 1), intermediate grade (grade 2), or high grade (grade 3 ) based on the proportion of solid growth and nuclear atypia, while the NEEC are high grade by definition. Using combinations of these risk factors, risk groups have been defined based 
on data from randomized trials, with each risk group having a distinct prognosis and adjuvant treatment recommendations have been determined for these risk groups (Table 1) [3].

\section{Treatment}

Women with EC are primarily treated with surgery, consisting of abdominal or laparoscopic hysterectomy and bilateral salpingo-oophorectomy, with or without lymph node evaluation. The indication for adjuvant treatment has been based on the presence of clinicopathological risk factors. Women with low or low-intermediate risk EC are treated with surgery alone $[4,5]$. Women with high-intermediate risk (HIR) EC usually receive adjuvant radiotherapy, mainly vaginal brachytherapy (VBT) [6-8]. Women with high-risk EC (HR), being at higher risk of recurrence, receive pelvic external beam radiotherapy (EBRT) with or without adjuvant chemotherapy. Especially in the case of substantial LVSI, EBRT is preferred over VBT alone to maximize pelvic nodal control in HIR EC [9-11]. The role of adjuvant chemotherapy, most often given in combination with EBRT, has been the subject of recent randomized trials, which showed increased relapse-free survival rates with combined adjuvant treatment at the cost of increased toxicity, and this is mainly recommended in stage III disease and for serous cancers $[12,13]$.

\section{Molecular-Genetic Characterization of EC and Molecular Risk Factors}

The extensive molecular-genetic characterization of endometrial cancer by the Cancer Genome Atlas Group (TCGA) has been pivotal in understanding the molecular pathways involved in endometrial cancer development and their prognostic implications. By full genomic analysis of 373 EC cases, 4 different molecular subclasses were identified based on mutation rates and somatic copy-number alterations (SCNA). The ultramutated subclass, characterized by mutations in the exonuclease domain of DNA polymerase-epsilon (POLE), is associated with a very favorable prognosis. The hypermutated subclass based on microsatellite instability (MSI) has been shown to have an intermediate prognosis. The copy-number low subclass with low mutation frequency (also called subclass with no specific molecular profile or NSMP) has also been associated with an intermediate prognosis. The copynumber high subclass, characterized by TP53 mutations, with mainly serous-type EC, has a very high degree of SCNAs and a low mutation rate and is associated with the most unfavorable prognosis $[14 \cdot \bullet]$. Several research groups have reproduced and validated the four TCGA subclasses in formalin-fixed, paraffin-embedded tissues in different EC cohorts by using their surrogate markers (Fig. 1a). These findings have led to a clinically available molecular classification tool for diagnosis and decision making $[11 \bullet, 15,16 \bullet, 17 \bullet, 18,19 \bullet]$.

The current guidelines for diagnosis and treatment of EC are based on the clinicopathologic factors, age, FIGO stage, histologic type and grade, myometrial invasion, and the presence of LVSI, but do not include molecular alterations. The current question is if and how these molecular risk factors can be used to guide adjuvant treatment. The purpose of this review was to provide an overview of the molecular risk factors involved in endometrial cancer in relation to their prognostic implications and potential therapeutic consequences.
Table 1 Risk groups in endometrial cancer as proposed by the ESMO-ESGO-ESTRO consensus guideline and related adjuvant treatment [3]

\begin{tabular}{lll}
\hline Risk group & Description & Current adjuvant treatments \\
\hline Low & FIGO stage IA EEC: grade 1-2, LVSI neg. & NAT \\
Intermediate & FIGO stage IB EEC: grade 1-2, LVSI neg. & VBT (NAT if age $<$ 60) \\
High-intermediate & FIGO stage IA/B EEC: grade 1-2, LVSI pos. & VBT (EBRT if stage IB LVSI pos.) \\
& FIGO stage IA EEC: grade 3, LVSI neg. & VBT (NAT if age $<$ 60) \\
& FIGO stage IA EEC: grade 3, LVSI pos. & EBRT (VBT if LNI neg.) \\
High & FIGO stage IB EEC: grade 3 & EBRT (VBT if LVSI neg. or LNI neg.) \\
& FIGO stage II EEC: grade 1-2, LVSI neg. & VBT \\
& FIGO stage II EEC: grade 1-2, LVSI pos. & EBRT +/ VBT boost \\
& FIGO stage II EEC: grade 3 or LVSI pos. & EBRT \\
& FIGO stage III EEC & EBRT $+C T^{*}$ \\
& FIGO stage IA SC/CC, LVSI neg. & VBT \\
& FIGO stage $\geq$ IB SC/CC & EBRT $+C T$ \\
\hline
\end{tabular}

$F I G O$, International Federation of Gynecology and Obstetrics 2009; EEC, endometrioid endometrial cancer; LVSI, lymph-vascular space invasion (neg.: negative, pos.: substantial LVSI); NAT, no adjuvant treatment; $V B T$, vaginal brachytherapy; $L N I$, lymph node involvement (surgical staged); $E B R T$, external beam radiotherapy; $C T$, chemotherapy; $S C$, serous carcinoma; $C C$, clear cell carcinoma

Italic: to be considered

*EBRT and chemotherapy either combined (PORTEC-3 and GOG 258 schedule) or sequentially 
a

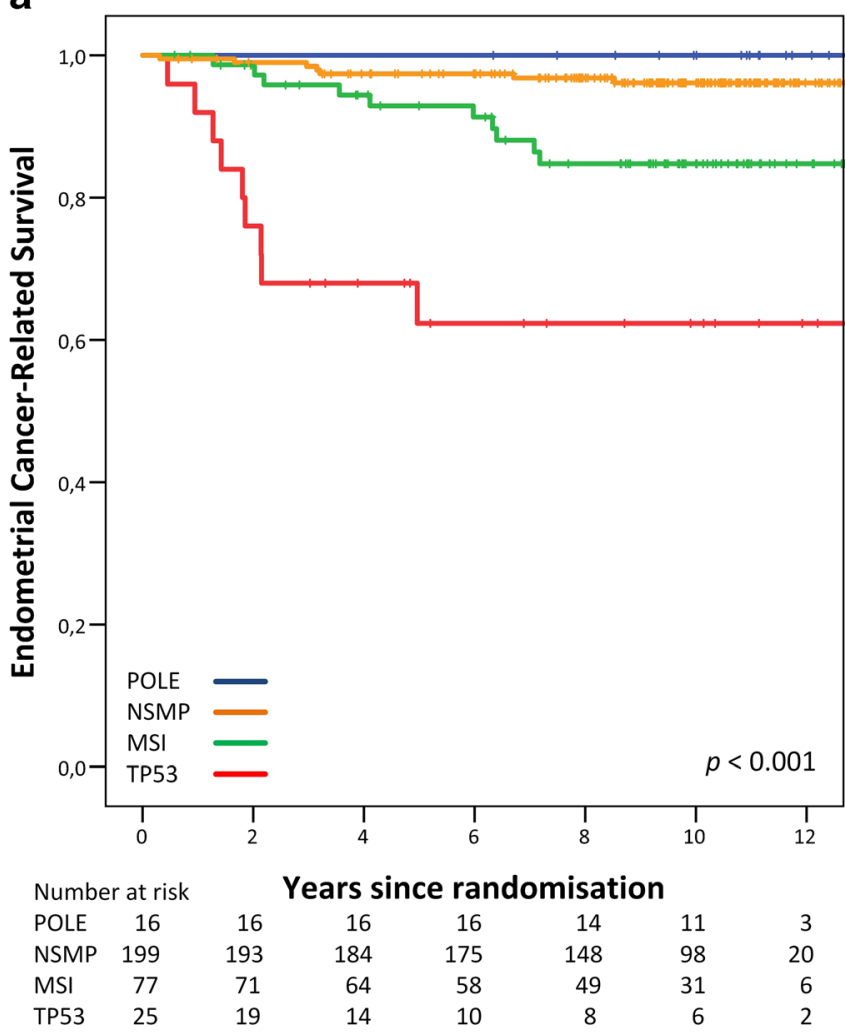

Fig. 1 a Endometrial cancer-related survival by 4 molecular subgroups in the PORTEC-2 trial for high-intermediate risk EC. b Total pelvic recurrence by unfavorable risk factors (LVSI, p53-mutant, or L1CAM expression). Reproduced from Wortman, B.G., et al., Ten-year results of the PORTEC-2 trial for high-intermediate risk endometrial carcinoma:

\section{Methods}

\section{Data Sources and Study Selection}

A PubMed search was performed including articles published between 1 January 2000 and 18 December 2018. Search terms included: endometrial cancer AND adjuvant radiotherapy OR adjuvant chemotherapy OR adjuvant chemoradiotherapy AND molecular pathways OR molecular risk factors. Preclinical research involving animal studies or endometrial cancer cell lines were excluded, as well as articles on uterine sarcomas. This search strategy resulted in 383 articles, which were subsequently selected on title, abstract, and full text. Additionally, review of the reference lists of these articles was performed and relevant papers were included.

\section{Results}

\section{Molecular Characterization}

Over the last few years, more specific knowledge of molecular alterations and molecular heterogeneity within EC has b

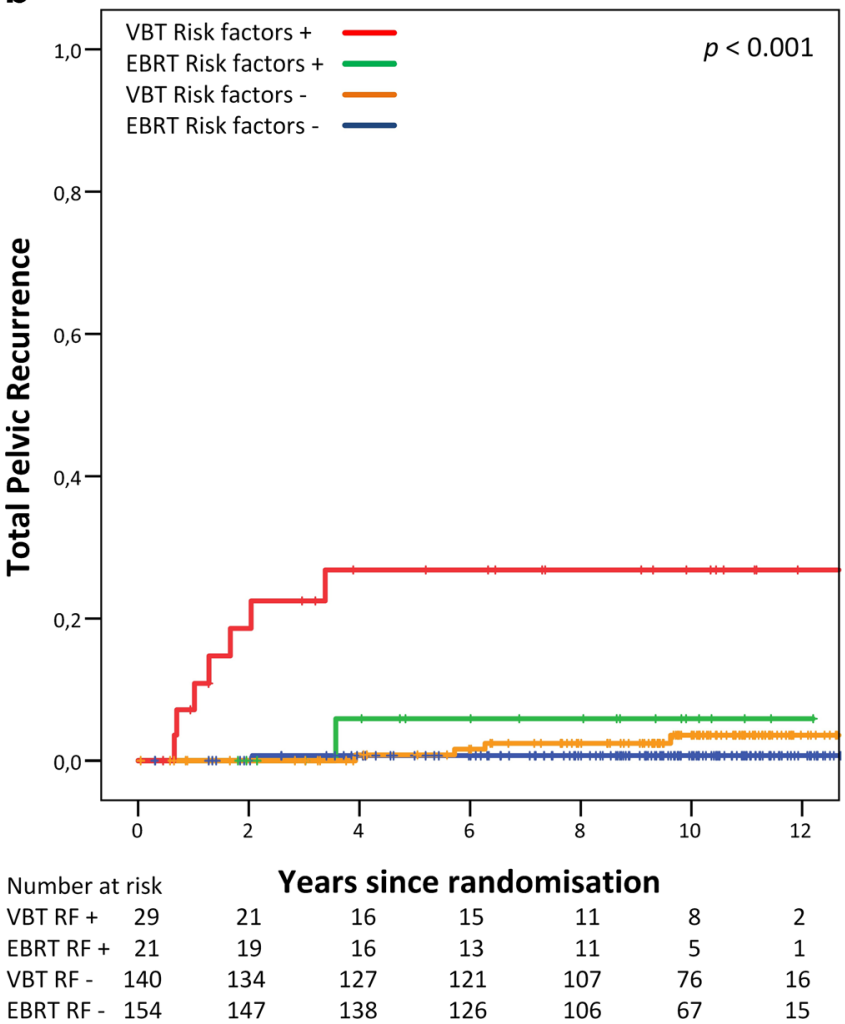

improving patient selection for adjuvant therapy. Br J Cancer, 2018. 119(9): p. 1067-1074, which is distributed under the terms of the Creative Commons Attribution 4.0 International License (http:// creativecommons.org/licenses/by/4.0/), courtesy of the authors

become available [14••]. The discoveries reported by TCGA have led to a great number of studies involving molecular characteristics and novel targets for therapy of EC (Table 2).

\section{POLE-Mutant Subclass}

Approximately 6 to $12 \%[14 \bullet \bullet, 16 \bullet, 17 \bullet, 18,19 \bullet, 20,21 \cdot]$ of all EC carries a pathogenic variant in the exonuclease domain of DNA polymerase epsilon (POLE) and are more frequently found in relatively younger women (median age 58-63.5 vs. 66-68.5) with lower BMI and higher grade endometrioid endometrial tumors compared to POLE wildtype EC $[21 \cdot, 22$, 23]. It is thought that $P O L E$ mutations impair the proofreading function during DNA replication that leads to an exceptionally high mutational burden. In POLE-mutant EC, an increased antitumor response by peritumoral and tumor-infiltrating CD8+ lymphocytes has been reported, most probably because the mutated DNA fragments act as neo-antigens that elicit a strong immune response [24-28]. In contrast to the aggressive appearance and high grade of POLE-mutant EC, they have consistently been shown to have an excellent prognosis with only an occasional relapse, both with and without adjuvant treatment. It has been suggested that their very favorable 
Table 2 Common molecular alterations in endometrial cancer.

\begin{tabular}{|c|c|c|c|c|}
\hline Molecular (pathway) alteration & $\begin{array}{l}\text { Frequency } \\
\text { in EC }\end{array}$ & Description & Prognosis & Potential targeted therapies \\
\hline POLE mutation & $6-12 \%$ & DNA repair & Excellent & PD-1/PD-L1 immune checkpoint inhibitors* \\
\hline MMRd & $20-40 \%$ & DNA repair & Intermediate & PD-1/PD-L1 immune checkpoint inhibitors* \\
\hline TP53 mutation & $9-29 \%$ & Tumor suppressor & Poor & PARPi; platinum derivatives \\
\hline L1CAM overexpression & $16-28 \%$ & Cell adhesion/signaling protein & Poor & $\mathrm{n} / \mathrm{a}^{*}$ \\
\hline ER/PR expression & $72-95 \%$ & Hormone receptors & Good & $\begin{array}{l}\text { Endocrine therapy } \\
\text { (with PI3K/mTOR inhibitors) }\end{array}$ \\
\hline Wnt- $\beta$-catenin pathway & $18-25 \%$ & Wnt signaling pathway & Intermediate & $\mathrm{n} / \mathrm{a}$ \\
\hline PI3K-AKT-mTOR pathway & $>80 \%$ & $\begin{array}{l}\mathrm{PI} 3 \mathrm{~K} / \mathrm{AKT} / \mathrm{mTOR} \\
\text { signaling pathway }\end{array}$ & Good-intermediate & PI3K, AKT, mTOR inhibitors \\
\hline HER2/Neu overexpression & $14-47 \%$ & Epidermal growth factor receptor & Poor & Monoclonal antibodies, protein kinase inhibitors \\
\hline ARIDIA mutation & $30-40 \%$ & Tumor suppressor & Good-intermediate & EZH2 inhibitors \\
\hline
\end{tabular}

$E C$, endometrial cancer; $n / a$, not available. For abbreviations see text

*The addition of PARP inhibitors (PARPi) has been explored for several molecular alterations, however evidence of efficacy is still limited

outcome is mainly based on the strong host immune response and that adjuvant (radio) therapy in early stage $P O L E$-mutant EC could be safely omitted [16•, 17•, 18, 19•, 21•, 22, 23, 29].

\section{MSI Subclass}

The TCGA has defined a microsatellite unstable (MSI) subclass; however, in clinical practice, mismatch repair deficiency (MMRd) is tested by immunohistochemistry of the MMR proteins. This subclass comprises between 20 and $40 \%$ of all EC [14••, 16•, 17•, 30, 31•, 32]. MMRd EC fails to express one or more of the MMR proteins MLH1, MSH2, MSH6, or PMS2, leading to the accumulation of mismatches, deletions, and microsatellite instability. The majority $(\sim 95 \%)$ of MMRd EC is MMR deficient due to $M L H-1$ promoter hypermethylation with subsequent loss of MLH1 protein expression. The remaining 3-6\% consists of MMRd EC cases due to either biallelic somatic mutations or germline defects in one of the MMR genes (Lynch syndrome) [33]. MMRd EC shows a similar increase in tumor-infiltrating lymphocytes as seen in $P O L E$-mutant EC, however, it is associated with negative prognostic factors such as higher histologic grade, presence of LVSI and with older age, and advanced stage (III/IV). MMRd EC has an intermediate prognosis in most studies [30, 31•, 32, 34-36].

\section{Copy-number High Subclass}

In this subclass characterized by a high number of somatic copy-number alterations and a relatively low mutation rate, 90\% of all tumors harbor somatic TP53 mutations. This subclass consists of serous and serous-like subtypes and is associated with unfavorable overall and progression-free survival. Both amplification of the human epidermal growth factor receptor 2 (Her-2/Neu) and homologous recombination deficiency $(\mathrm{HRd})$ are frequent molecular alterations in this subclass $[14 \bullet \bullet, 17 \bullet, 37 \bullet]$.

\section{Copy-number Low Subclass}

Within the copy-number low (CNL) subclass, no specific molecular driver could be identified. This large subclass is characterized by a low mutational burden, comprises most endometrioid-type cancers of low to intermediate grade and is associated with an intermediate prognosis. Frequent molecular alterations in this subclass are CTNNB1 mutations ( $52 \%$ ) and PI3K pathway alterations [14॰•]. The CNL subclass contains a heterogeneous set of tumors, which complicates the distinction of favorable or unfavorable tumors. A subgroup with amplification of 1q32.1 has been reported to be associated with impaired cancer-specific and recurrence-free survival [38]. On the contrary, among all grade 3 ECs, the CNL subclass is associated with a favorable prognosis as compared with the other grade 3 ECs [39॰].

\section{Additional Molecular Characteristics and Pathways}

\section{Endocrine Receptors}

Expression of estrogen (ER) and progestogen (PR) receptors is common in EC, especially in EEC, with a reported frequency of $72-81 \%$ [40]. ER and PR can stimulate or inhibit the transcription of several genes and are associated with lowgrade tumors, more favorable tumor histology, and favorable prognosis. Loss of ER or PR expression is related to higher grade tumors and impaired disease-free survival [41, 42]. Targeting the endocrine receptors by hormonal therapy is used for women with low-grade early stage EC that wish to preserve fertility and also for those with low-grade metastatic EC [3]. Hormonal therapy in women with grade 1 metastatic 
EEC, especially those with lung or oligometastasis, can provide durable responses [43].

\section{PI3K-AKT-mTOR Pathway}

The PI3K-AKT-mTOR pathway is essential for regulating diverse cellular responses including cell growth and survival and is altered in over $80 \%$ of all ECs [14••, 44, 45]. Somatic loss of PTEN, a tumor suppressor gene, is the most frequent alteration in this pathway. Other common alterations are mutations in the PIK3CA, PIK3R1, and KRAS genes. While loss of $P T E N$ is often correlated with endometrioid histology, low tumor grade, and favorable prognosis, PIK3CA is associated with high tumor grade. As PI3K-AKT-mTOR is the most common pathway alteration in EC, many therapeutic options have been explored. Targeted therapy of the PI3K pathway by using PI3K inhibitors as single agents has not shown satisfactory results, and novel dual PI3K-mTOR, mTOR, and AKT inhibitors are being investigated. In previous studies, it has been suggested that PI3K-AKT-mTOR pathway inhibition suppresses resistance to endocrine therapy in several solid tumors, including EC [45-48]. Promising results have been described for combined treatment with PI3K inhibition and endocrine therapy, and the combination of everolimus and letrozole has been shown to lead to $53 \%$ response rates in chemo-naïve patients with recurrent EC [49, 50•].

Metformin has also been linked to regulation of PI3KAKT-mTOR signaling and activates the AMP protein kinase (AMPK) pathway, which by several processes, regulates cell growth via suppression of mTOR. The clinical activity of metformin has been investigated; however, a recent randomized placebo-controlled phase 3 window trial in women with endometrial hyperplasia and EEC did not show reduction of tumor proliferation $[46,51]$.

\section{ARID1A}

Mutation of the AT-rich interactive domain 1A (ARIDIA) gene has been found in approximately $30-40 \%$ of EEC and only in $0-7 \%$ of serous EC [14••, 52, 53]. ARID1A is involved in the switch/sucrose non-fermentable (SWI/SNF) chromatin remodeling complex and encodes a nuclear protein, BAF250a, that is involved in essential cell-cycle control and DNA damage repair pathways. Loss of BAF250a protein expression can result in cell proliferation and malignant progression of precursor lesions. A possibility for targeted therapy in ARID1A-mutated EC by inhibition of its downregulator EZH2, a methyltransferase enzyme, has been described, however, no (randomized) clinical trials have been performed so far [52-55]. Other suggestions for targeted therapy in this particular subgroup are PI3K inhibitors, as previous studies have described a crosstalk between ARID1A and the PI3KAKT-mTOR pathway [54].

\section{ERBB2 Receptors}

The epithelial growth factor (EGF) system consists of four receptors EGFR, HER-2/Neu, HER-3, and HER-4. HER-2/ Neu amplification and/or overexpression are most frequently found in serous EC, with wide variations in reported frequencies of amplification and overexpression, which range between $14-80 \%$ and $21-47 \%$, respectively [56]. This can be explained by different testing or scoring methods used and test result interpretation, as currently, no standardized testing methods for HER-2/Neu in EC exist. In order to successfully perform clinical trials of HER-2/Neu-targeted therapy, identification of patients that might benefit the most from this therapy is a key aspect of eligibility. Previous studies have suggested that the HER-2/Neu receptor might be a potential target for therapy, however, studies using HER-2/Neu inhibitors as monotherapy have had disappointing results [56-59]. In a recent study, the combination of trastuzumab with carboplatin and paclitaxel chemotherapy in HER-2/Neu-positive serous carcinomas resulted in a prolonged median progression-free survival of 13 months compared to 8 months in the carboplatin-paclitaxel alone group $(p=0.005)$ [60 $]$.

\section{Wnt-ß-catenin Pathway}

Molecular alterations in the Wnt-ß-catenin pathway are found in approximately $52 \%$ of EC within the NSMP subclass and approximately $18-25 \%$ of ECs in general [14••, 44, 61, 62]. ßcatenin acts as a transcriptional factor in the Wnt-pathway that regulates gene transcription and development. Estrogens can induce Wnt/B-catenin signaling, which may result in growth stimulation of endometrial hyperplasia and well-differentiated EC, while progesterone can inhibit this process [63]. In the normal situation, $\beta$-catenin levels are kept low through degradation by the ubiquitin-proteasome pathway [64]. In ß-catenin (CTNNB1)-mutated EC, the Wnt signaling pathway is activated by nuclear accumulation of $\beta$-catenin that results in $\mathrm{EC}$ progression and abnormal expression of cell proliferation and progression genes. CTNNB1 exon 3 mutation is associated with decreased overall survival $[62,65]$.

\section{Programmed Cell Death-1/Ligand-1}

Both POLE-mutant and MMRd ECs contain a high mutational burden which elicits an increased immune response by tumor infiltrating lymphocytes (TIL). It has been shown that hypermutated tumors harbor higher neoantigen loads, which are associated with increased immune response by cytotoxic CD8+ TILs. In a response to this, the tumor upregulates immune-inhibitory molecules like PD-1/PD-L1 and others $[25,26]$. Increased PD-1 and PD-L1 expression have made these tumors attractive for immune checkpoint inhibitors acting against PD-1 receptor or its ligand PD-L1 [25, 28•, 29, 36, 
$66,67 \bullet$. Previous trials have confirmed this hypothesis and have shown remarkable clinical responses to nivolumab, pembrolizumab, and lenvatinib in women with recurrent or metastatic hypermutated tumors, including EC [68-70]. Pembrolizumab has been approved by the FDA for the treatment of women with metastatic MMRd EC.

\section{Homologous Recombination Deficiency}

A relative frequent DNA repair defect, $\mathrm{HRd}$, has recently been observed especially in the TP53-mutated or serous-like subclass. Homologous recombination is essential for repair of DNA double-strand breaks, which is mediated by (among others) BRCA1 and BRCA2 proteins. In a study of $24 \mathrm{EC}$, HRd was analyzed by detecting RAD51 foci after irradiation of tissue samples, and HRd was found in $24 \%$ of EC. All 6 HRd EC were non-endometrioid cancers, especially serous type EC, and in $67 \%$ of all NEEC, HRd was found. In 5 of the 6 HRd EC, a molecular basis for HRd could be identified by somatic copy-number alterations in the BRCA core or related genes. Targeting HRd in EC by using platinum-based chemotherapy and/or PARP inhibitors seem promising therapeutic options [37•].

PARP, or poly (ADP-ribose) polymerase is involved in DNA damage detection and generation of poly (ADPribose) chains. These chains facilitate chromatin remodeling and DNA repair. Loss of PARP results in persistent singlestrand DNA breaks and eventually in double-strand DNA breaks. Normally, DSBs are repaired by homologous recombination or other repair mechanisms such as non-homologous end joining. Tumor cells with either loss of PARP or with HRd alone are still viable, however, in case of simultaneous inhibition of both repair pathways, accumulation of DSBs leads to cell death $[71 \bullet, 72]$. Objective responses to PARP inhibitors (PARPi) have been reported in women with HRd (BRCA mutant or BRCA wildtype) ovarian carcinoma and in BRCA-mutated breast cancer [73, 74]. Another useful consequence of PARPi is the increase of neo-antigen expression caused by the accumulating DNA damage, resulting in an increased anti-tumor immune response, suggesting that a combination of PARPi with immune checkpoint inhibitors might be an effective and even synergistic treatment. Most promising would be the use of this combination in the subgroup of patients with hypermutated ECs with high TIL counts (POLE-mutant or MMRd EC) and those with HRd $\mathrm{EC}[37 \cdot, 71 \cdot, 72]$.

\section{Limitations of the Current Guidelines}

The molecular discoveries by the TCGA have provided new insights into the carcinogenesis and clinical behavior of endometrial cancer. EC within the subclass with POLE mutation have shown an excellent prognosis, even without further adjuvant therapy, while EC with unfavorable molecular alterations have shown an increased risk of locoregional recurrence and distant spread of the disease. These molecular risk factors have not yet been included in clinical guidelines [3]. Combining molecular features and clinicopathological risks factors into a stratification model holds promise to guide surgery, adjuvant treatment, and disease surveillance, which results in more individualized adjuvant treatment and reduction of both under and overtreatment $[16 \bullet, 17 \cdot]$. Furthermore, a combined assessment can lead to a more objective approach of EC classification, compared to the current histology-based classification, which has its limitations as inter-observer variability $[75,76]$. Apart from this, other strategies such as the molecular assessment of endometrial biopsies, which has shown high concordance with the final hysterectomy specimen, could guide both (pre-)surgical treatment decisions such as the extent of surgical staging $[77,78]$.

\section{Integrated Molecular Risk Profile and Adjuvant Treatment}

In a comprehensive translational research project, $834 \mathrm{HIR}$ EC samples of the pooled PORTEC-1 and 2 biobank were analyzed, and the TCGA subclasses and other molecular risk factors such as CTNNB1 exon 3 mutations were combined with clinicopathologic factors such as presence of LVSI and with L1-cell adhesion molecule (L1CAM) overexpression [9, $10,17 \cdot$. L1CAM is a membrane glycoprotein with an important role in tumor cell adhesion and migration. L1CAM overexpression $(>10 \%)$ on immunohistochemistry is reported in approximately $16-28 \%$ of EC and is associated with frequent TP53 mutation, non-endometrioid histology, histological grade 3, LVSI, and with an increased risk of locoregional and distant spread. It is independently related to decreased overall and relapse-free survival [79-82, 83• 84].

Combining all these risk factors resulted in 3 subgroups within HIR EC with favorable, intermediate and unfavorable outcomes. The favorable subgroup with either POLE-mutant EC or with absence of other risk factors had an excellent prognosis, for which it was suggested that adjuvant treatment could safely be omitted; the unfavorable subgroup, strongly associated with the risk of locoregional and distant spread, with either TP53-mutation, L1CAM overexpression, or substantial LVSI, for which more intensive adjuvant treatment by EBRT seems justified (Fig. 1b), and an intermediate subgroup with either MMRd or CTNNB1 mutation [11•]. For this intermediate group, most benefit of the standard treatment VBT is expected. This molecular-integrated risk model with corresponding consequences for adjuvant treatment is currently being investigated in the international, multicenter randomized controlled PORTEC-4a trial, of which recent evaluation 
of the pilot phase showed feasible trial logistics and a satisfactory patient acceptance rate $[85,86]$.

\section{Conclusions and Future Directions}

Molecular alterations are frequently found in endometrial cancer and increasing knowledge on their prognostic significance and possible therapeutic options has been gained. Trials investigating (adjuvant) treatment based on molecular alterations are ongoing for women with high-intermediate risk, high risk, and recurrent or metastatic EC. More evidence on selecting adjuvant radiotherapy, chemotherapy, and targeted therapies based on molecular alterations in EC are needed and results of ongoing and future trials investigating adjuvant and targeted therapies for EC are eagerly awaited.

\section{Compliance with Ethical Standards}

Conflict of Interest Bastiaan G. Wortman declares that he has no conflict of interest.

Remi A. Nout declares that he has no conflict of interest.

Tjalling Bosse declares that he has no conflict of interest.

Carien L. Creutzberg is supported by a clinical trial grant from the Dutch Cancer Society for the PORTEC-4a Trial.

Human and Animal Rights and Informed Consent This article does not contain any studies with human or animal subjects performed by any of the authors.

Open Access This article is distributed under the terms of the Creative Commons Attribution 4.0 International License (http:// creativecommons.org/licenses/by/4.0/), which permits unrestricted use, distribution, and reproduction in any medium, provided you give appropriate credit to the original author(s) and the source, provide a link to the Creative Commons license, and indicate if changes were made.

\section{References}

Papers of particular interest, published recently, have been highlighted as:

- Of importance

•- Of major importance

1. IKNL. Dutch Cancer Registry. Available at: http://www. cijfersoverkanker.nl. Accessed January 8, 2019.

2. Siegel RL, Miller KD, Jemal A. Cancer statistics, 2019. CA Cancer J Clin. 2019;69(1):7-34.

3. Colombo N, et al. ESMO-ESGO-ESTRO Consensus Conference on Endometrial Cancer: diagnosis, treatment and follow-up. Ann Oncol. 2016;27(1):16-41.

4. Creutzberg CL, et al. Surgery and postoperative radiotherapy versus surgery alone for patients with stage- 1 endometrial carcinoma: multicentre randomised trial. PORTEC Study Group. Post operative radiation therapy in endometrial carcinoma. Lancet. 2000;355(9213):1404-11.
5. Keys HM, et al. A phase III trial of surgery with or without adjunctive external pelvic radiation therapy in intermediate risk endometrial adenocarcinoma: a Gynecologic Oncology Group study. Gynecol Oncol. 2004;92(3):744-51.

6. Blake $\mathrm{P}$, et al. Adjuvant external beam radiotherapy in the treatment of endometrial cancer (MRC ASTEC and NCIC CTG EN.5 randomised trials): pooled trial results, systematic review, and meta-analysis. Lancet. 2009;373(9658):137-46.

7. Nout RA, et al. Vaginal brachytherapy versus pelvic external beam radiotherapy for patients with endometrial cancer of highintermediate risk (PORTEC-2): an open-label, non-inferiority, randomised trial. Lancet. 2010;375(9717):816-23.

8. Sorbe B, et al. External pelvic and vaginal irradiation versus vaginal irradiation alone as postoperative therapy in medium-risk endometrial carcinoma-a prospective randomized study. Int J Radiat Oncol Biol Phys. 2012;82(3):1249-55.

9. Guntupalli SR, et al. Lymphovascular space invasion is an independent risk factor for nodal disease and poor outcomes in endometrioid endometrial cancer. Gynecol Oncol. 2012;124(1): 31-5.

10. Bosse T, et al. Substantial lymph-vascular space invasion (LVSI) is a significant risk factor for recurrence in endometrial cancer-a pooled analysis of PORTEC 1 and 2 trials. Eur J Cancer. 2015;51(13):1742-50.

11. Wortman BG, et al. Ten-year results of the PORTEC-2 trial for high-intermediate risk endometrial carcinoma: improving patient selection for adjuvant therapy. Br J Cancer. 2018;119(9):1067-74 This study reports on the long-term results of the PORTEC-2 trial that investigates VBT compared to EBRT in women with high-intermediate risk $\mathrm{EC}$ with a subanalysis on risk factors for improved adjuvant treatment selection in EC.

12. Hogberg T, et al. Sequential adjuvant chemotherapy and radiotherapy in endometrial cancer-results from two randomised studies. Eur J Cancer. 2010;46(13):2422-31.

13. de Boer SM, et al. Adjuvant chemoradiotherapy versus radiotherapy alone for women with high-risk endometrial cancer (PORTEC3): final results of an international, open-label, multicentre, randomised, phase 3 trial. Lancet Oncol. 2018;19(3):295-309.

14.• Kandoth C, et al. Integrated genomic characterization of endometrial carcinoma. Nature. 2013;497(7447):67-73 In this study, four different molecular subclasses in EC with each a distinct prognosis are defined based on extensive genomic characterisation of endometrial cancer samples.

15. Stelloo E, et al. Refining prognosis and identifying targetable pathways for high-risk endometrial cancer; a TransPORTEC initiative. Mod Pathol. 2015;28(6):836-44.

16. Talhouk A, et al. A clinically applicable molecular-based classification for endometrial cancers. Br J Cancer. 2015;113(2):299-310 Analysis of the four TCGA subclasses by their surrogate markers in endometrial cancer that led to a molecular classification tool for the clinic.

17. Stelloo E, et al. Improved risk assessment by integrating molecular and clinicopathological factors in early-stage endometrial cancercombined analysis of the PORTEC cohorts. Clin Cancer Res. 2016;22(16):4215-24 In this study, the four TCGA subclasses are analyzed by their surrogate markers in a large $\mathrm{EC}$ cohort and integrated in a molecular integrated risk profile.

18. Talhouk A, et al. Confirmation of ProMisE: a simple, genomicsbased clinical classifier for endometrial cancer. Cancer. 2017;123(5):802-13.

19. Kommoss S, et al. Final validation of the ProMisE molecular classifier for endometrial carcinoma in a large population-based case series. Ann Oncol. 2018;29(5):1180-8 In this study, the molecular classification tool is validated. 
20. Church DN, et al. DNA polymerase epsilon and delta exonuclease domain mutations in endometrial cancer. Hum Mol Genet. 2013;22(14):2820-8.

21. Church DN, et al. Prognostic significance of POLE proofreading mutations in endometrial cancer. J Natl Cancer Inst. 2015;107(1): 402 In this study, survival analysis of POLE mutant EC in two large randomized controlled trials and three additional cohorts of early stage $\mathrm{EC}$ is performed.

22. Billingsley CC, et al. Prognostic significance of POLE exonuclease domain mutations in high-grade endometrioid endometrial cancer on survival and recurrence: a subanalysis. Int J Gynecol Cancer. 2016;26(5):933-8.

23. McConechy MK, et al. Endometrial carcinomas with POLE exonuclease domain mutations have a favorable prognosis. Clin Cancer Res. 2016;22(12):2865-73.

24. Hussein YR, et al. Clinicopathological analysis of endometrial carcinomas harboring somatic POLE exonuclease domain mutations. Mod Pathol. 2014;28(4):505-14.

25. Howitt BE, et al. Association of polymerase e-mutated and microsatellite-instable endometrial cancers with neoantigen load, number of tumor-infiltrating lymphocytes, and expression of PD1 and PD-L1. JAMA Oncol. 2015;1(9):1319-23.

26. van Gool IC, et al. POLE proofreading mutations elicit an antitumor immune response in endometrial cancer. Clin Cancer Res. 2015;21(14):3347-55.

27. Bakhsh S, et al. Histopathological features of endometrial carcinomas associated with POLE mutations: implications for decisions about adjuvant therapy. Histopathology. 2016;68(6):916-24.

28. Eggink FA, et al. Immunological profiling of molecularly classified high-risk endometrial cancers identifies POLE-mutant and microsatellite unstable carcinomas as candidates for checkpoint inhibition. Oncoimmunology. 2017;6(2):e1264565 This study investigates the rationale of check point inhibition strategies in POLE mutant and MSI EC.

29. Van Gool IC, et al. Adjuvant treatment for POLE proofreading domain-mutant cancers: sensitivity to radiotherapy, chemotherapy, and nucleoside analogues. Clin Cancer Res. 2018;24(13):3197203.

30. Zighelboim I, et al. Microsatellite instability and epigenetic inactivation ofMLH1 and outcome of patients with endometrial carcinomas of the endometrioid type. J Clin Oncol. 2007;25(15):2042-8.

31. McMeekin DS, et al. Clinicopathologic significance of mismatch repair defects in endometrial cancer: An NRG Oncology/ Gynecologic Oncology Group Study. J Clin Oncol. 2016;34(25): 3062-8 This study analyzes the relationship between tumor MMR status and outcomes and response to adjuvant therapy.

32. Cosgrove CM, et al. Epigenetic silencing of MLH1 in endometrial cancers is associated with larger tumor volume, increased rate of lymph node positivity and reduced recurrence-free survival. Gynecol Oncol. 2017;146(3):588-95.

33. Leenen $\mathrm{CH}$, et al. Prospective evaluation of molecular screening for Lynch syndrome in patients with endometrial cancer $</=70$ years. Gynecol Oncol. 2012;125(2):414-20.

34. MacDonald ND, et al. Frequency and prognostic impact of microsatellite instability in a large population-based study of endometrial carcinomas. Cancer Res. 2000;60:1750-2.

35. Pijnenborg JM, et al. Defective mismatch repair and the development of recurrent endometrial carcinoma. Gynecol Oncol. 2004;94(2):550-9.

36. Kim SR, et al. Does MMR status in endometrial cancer influence response to adjuvant therapy? Gynecol Oncol. 2018;151(1):76-81.

37. de Jonge MM, et al. Frequent homologous recombination deficiency in high-grade endometrial carcinomas. Clin Cancer Res. 2018; This study investigates the prevalence of homologous recombination deficiency in high-risk EC, its association with histopathologic and molecular characteristics and explores therapeutic options.

38. Depreeuw J, et al. Amplification of 1q32.1 refines the molecular classification of endometrial carcinoma. Clin Cancer Res. 2017;23(23):7232-41.

39. Bosse T, et al. Molecular classification of grade 3 endometrioid endometrial cancers identifies distinct prognostic subgroups. Am J Surg Pathol. 2018;42(5):561-8 In this study, the four TCGA subgroups are analyzed by their surrogate markers in a subgroup of grade 3 EEC.

40. van der Putten LJM, et al. Added value of estrogen receptor, progesterone receptor, and $\mathrm{L} 1$ cell adhesion molecule expression to histology-based endometrial carcinoma recurrence prediction models: an ENITEC collaboration study. Int J Gynecol Cancer. 2018;28(3):514-23.

41. Jongen V, et al. Expression of estrogen receptor-alpha and -beta and progesterone receptor-A and -B in a large cohort of patients with endometrioid endometrial cancer. Gynecol Oncol. 2009;112(3): 537-42.

42. Salvesen HB, Haldorsen IS, Trovik J. Markers for individualised therapy in endometrial carcinoma. Lancet Oncol. 2012;13(8):e35361.

43. Ethier JL, et al. Is hormonal therapy effective in advanced endometrial cancer? A systematic review and meta-analysis. Gynecol Oncol. 2017;147(1):158-66.

44. Murali R, Soslow RA, Weigelt B. Classification of endometrial carcinoma: more than two types. Lancet Oncol. 2014;15(7):e268 78.

45. Yuan TL, Cantley LC. PI3K pathway alterations in cancer: variations on a theme. Oncogene. 2008;27(41):5497-510.

46. Slomovitz BM, Coleman RL. The PI3K/AKT/mTOR pathway as a therapeutic target in endometrial cancer. Clin Cancer Res. 2012;18(21):5856-64.

47. Garcia-Dios DA, et al. High-throughput interrogation of PIK3CA, PTEN, KRAS, FBXW7 and TP53 mutations in primary endometrial carcinoma. Gynecol Oncol. 2013;128(2):327-34.

48. Myers AP. New strategies in endometrial cancer: targeting the $\mathrm{PI} 3 \mathrm{~K} / \mathrm{mTOR}$ pathway-the devil is in the details. Clin Cancer Res. 2013;19(19):5264-74.

49. Slomovitz BM, et al. Phase II study of everolimus and letrozole in patients with recurrent endometrial carcinoma. J Clin Oncol. 2015;33(8):930-6.

50. Slomovitz BM, et al. GOG 3007, a randomized phase II (RP2) trial of everolimus and letrozole (EL) or hormonal therapy (medroxyprogesterone acetate/tamoxifen, PT) in women with advanced, persistent or recurrent endometrial carcinoma (EC): A GOG Foundation study. Gynecol Oncol. 2018;149:2 This randomized phase II trial investigates the effect of the combination of Everolimus and Letrozole in women with recurrent EC.

51. Kitson $\mathrm{S}$, et al. PRE-surgical metformin in uterine malignancy (PREMIUM): a multi-center, randomized double-blind, placebocontrolled phase 3 trial. Clin Cancer Res. 2018.

52. Wiegand KC, et al. ARID1 A mutations in endometriosis-associated ovarian carcinomas. N Engl J Med. 2010;363(16):1532-43.

53. Guan B, Wang TL, Shih Ie M. ARID1A, a factor that promotes formation of SWI/SNF-mediated chromatin remodeling, is a tumor suppressor in gynecologic cancers. Cancer Res. 2011;71(21):671827.

54. Wu RC, Wang TL, Shih Ie M. The emerging roles of ARID1A in tumor suppression. Cancer Biol Ther. 2014;15(6):655-64.

55. Yen TT, et al. Loss of ARID1A expression in endometrial samplings is associated with the risk of endometrial carcinoma. Gynecol Oncol. 2018;150(3):426-31.

56. Buza N, Roque DM, Santin AD. HER2/neu in endometrial cancer: a promising therapeutic target with diagnostic challenges. Arch Pathol Lab Med. 2014;138(3):343-50. 
57. Holbro T. The ErbB receptors and their role in cancer progression. Exp Cell Res. 2003;284(1):99-110.

58. Grushko TA, et al. An exploratory analysis of HER-2 amplification and overexpression in advanced endometrial carcinoma: a Gynecologic Oncology Group study. Gynecol Oncol. 2008;108(1):3-9.

59. Fleming GF, et al. Phase II trial of trastuzumab in women with advanced or recurrent, HER2-positive endometrial carcinoma: a Gynecologic Oncology Group study. Gynecol Oncol. 2010;116(1):15-20.

60. Fader AN, et al. Randomized phase ii trial of carboplatin-paclitaxel versus carboplatin-paclitaxel-trastuzumab in uterine serous carcinomas that overexpress human epidermal growth factor receptor 2/neu. J Clin Oncol. 2018;36(20):2044-51 This randomized controlled phase 2 trial investigates the addition of trastuzumab to carboplatin-paclitaxel in uterine serous carcinomas with HER2/neu positivity.

61. Machin P, et al. CTNNB1 mutations and $\beta$-catenin expression in endometrial carcinomas. Hum Pathol. 2002;33(2):206-12.

62. Kurnit KC, et al. CTNNB1 (beta-catenin) mutation identifies low grade, early stage endometrial cancer patients at increased risk of recurrence. Mod Pathol. 2017;30(7):1032-41.

63. Wang Y, et al. Wnt/B-catenin and sex hormone signaling in endometrial homeostasis and cancer. Oncotarget. 2010;1(7):674-84.

64. Scholten AN, et al. Nuclear beta-catenin is a molecular feature of type I endometrial carcinoma. J Pathol. 2003;201(3):460-5.

65. Liu Y, et al. Clinical significance of CTNNB1 mutation and Wnt pathway activation in endometrioid endometrial carcinoma. J Natl Cancer Inst. 2014;106(9).

66. Le DT, et al. PD-1 blockade in tumors with mismatch-repair deficiency. N Engl J Med. 2015;372(26):2509-20.

67. Le DT, et al. Mismatch repair deficiency predicts response of solid tumors to PD-1 blockade. Science. 2017;357(6349):409-13 This study evaluates the efficacy of PD-1 blockade in patients with advanced MMRd cancers.

68. Mehnert JM, et al. Immune activation and response to pembrolizumab in POLE-mutant endometrial cancer. J Clin Invest. 2016;126(6):2334-40.

69. Santin AD, et al. Regression of chemotherapy-resistant polymerase epsilon (POLE) ultra-mutated and MSH6 hyper-mutated endometrial tumors with nivolumab. Clin Cancer Res. 2016;22(23):56827.

70. Makker V, et al. Lenvatinib plus pembrolizumab in patients with advanced endometrial cancer: an interim analysis of a multicentre, open-label, single-arm, phase 2 trial. Lancet Oncol. 2019;20(5): 711-8 This multicenter phase 2 trial investigates the effect of lenvantinib combined with pembrolizumab in patients with previously treated and advanced EC.

71. Lord CJ, Ashworth A. PARP inhibitors: synthetic lethality in the clinic. Science. 2017;355(6330):1152-8 This study discusses the current knowledge of PARP inhibitors and potential ways to maximize their effectiveness.

72. Rimar KJ, et al. The emerging role of homologous recombination repair and PARP inhibitors in genitourinary malignancies. Cancer. 2017;123(11):1912-24.
73. O'Sullivan CC, et al. Beyond breast and ovarian cancers: PARP inhibitors for BRCA mutation-associated and BRCA-like solid tumors. Front Oncol. 2014;4:42.

74. Swisher EM, et al. Rucaparib in relapsed, platinum-sensitive highgrade ovarian carcinoma (ARIEL2 Part 1): an international, multicentre, open-label, phase 2 trial. Lancet Oncol. 2017;18(1): 75-87.

75. Khalifa MA, et al. Slide review in gynecologic oncology ensures completeness of reporting and diagnostic accuracy. Gynecol Oncol. 2003;90(2):425-30.

76. Manion E, Cohen MB, Weydert J. Mandatory second opinion in surgical pathology referral material: clinical consequences of major disagreements. Am J Surg Pathol. 2008;32:732-7.

77. Stelloo E, et al. High concordance of molecular tumor alterations between pre-operative curettage and hysterectomy specimens in patients with endometrial carcinoma. Gynecol Oncol. 2014;133(2):197-204.

78. Talhouk A, et al. Molecular classification of endometrial carcinoma on diagnostic specimens is highly concordant with final hysterectomy: earlier prognostic information to guide treatment. Gynecol Oncol. 2016;143(1):46-53.

79. Fogel M, et al. L1 expression as a predictor of progression and survival in patients with uterine and ovarian carcinomas. Lancet. 2003;362(9387):869-75.

80. Zeimet AG, et al. L1CAM in early-stage type I endometrial cancer: results of a large multicenter evaluation. J Natl Cancer Inst. 2013;105(15):1142-50.

81. Bosse T, et al. L1 cell adhesion molecule is a strong predictor for distant recurrence and overall survival in early stage endometrial cancer: pooled PORTEC trial results. Eur J Cancer. 2014;50(15): 2602-10.

82. Geels YP, et al. L1CAM expression is related to non-endometrioid histology, and prognostic for poor outcome in endometrioid endometrial carcinoma. Pathol Oncol Res. 2016;22(4):863-8.

83. van der Putten LJ, et al. L1CAM expression in endometrial carcinomas: an ENITEC collaboration study. Br J Cancer. 2016;115(6): 716-24 This study analyzes the value of L1CAM overexpression in a large EC cohort and its association with prognostic features.

84. Kommoss F, et al. L1CAM: amending the "low-risk" category in endometrial carcinoma. J Cancer Res Clin Oncol. 2017;143(2): 255-62.

85. Wortman BG, et al. Molecular-integrated risk profile to determine adjuvant radiotherapy in endometrial cancer: Evaluation of the pilot phase of the PORTEC-4a trial. Gynecol Oncol. 2018;151(1):69-75.

86. ISRCTN-registry. PORTEC-4a: Randomised trial of standard or molecular profilebased recommendation for radiotherapy after surgery for women with early stage endometrial cancer. http://www. isrctn.com/ISRCTN11659025, 2016. Accessed February 19, 2019.

Publisher's Note Springer Nature remains neutral with regard to jurisdictional claims in published maps and institutional affiliations. 